\title{
Hepatic Lipid Droplets
}

\author{
ISOLATION, MORPHOLOGY AND COMPOSITION
}

\author{
By RICHARD P. DIAUGUSTINE, JOAN-MARIE SCHAEFER* and JAMES R. FOUTS \\ National Institute of Environmental Health Sciences, National Institutes of Health, \\ P.O. Box 12233, Research Triangle Park, N.C. 27709, U.S.A.
}

(Received 3 July 1972)

\begin{abstract}
The floating lipid layer isolated by centrifugation of rat liver was examined for composition and ultrastructure. It was chiefly composed of triglycerides and cholesterol esters plus much smaller amounts of free cholesterol, diglycerides, phospholipid and protein. No free fatty acids were detected. The triglyceride and cholesterol ester fractions consisted mostly of esters of linoleic acid, oleic acid and palmitic acid. Electron micrographs of the floating lipid layer revealed numerous spherical osmiophilic droplets having a mean diameter of $0.5-2 \mu \mathrm{m}$ with a very-thin dense outer coat. Similar structures were observed as organelles in electron micrographs of the intact liver cell. The amount of triglyceride in the layer decreased in rats starved for $72 \mathrm{~h}$, but pellet triglyceride (homogenate minus the floating lipid layer) was unchanged. These results suggest that the floating lipid layer is the representative in vitro of lipid-rich organelles which probably function as a depot form of hepatic-cell neutral lipid.
\end{abstract}

Reports of the presence of large homogeneous osmiophilic droplets in the mammalian hepatocyte have been reviewed by other investigators (Novikoff \& Essner, 1960; Rouiller \& Jézéquel, 1963). These droplets are about $0.5-2 \mu \mathrm{m}$ in diameter, appear to occur randomly in the cytoplasm and have been assumed, on a cytochemical basis, to consist predominantly of neutral lipids. Smaller osmiophilic droplets, usually less than $0.2 \mu \mathrm{m}(2000 \AA)$ in diameter, have been labelled as hepatic 'liposomes' and are usually observed in Golgi vesicles and in the cisternae of the endoplasmic reticulum, especially after pretreatment in vivo with agents that induce a fatty liver (Novikoff et al., 1964; Baglio \& Farber, 1965; Stein \& Stein, 1965, 1967; Schlunk \& Lombardi, 1967). The smaller osmiophilic droplets, or hepatic liposomes are present in the sedimented fraction after centrifugation of liver homogenates (Schlunk \& Lombardi, 1967). Contrary to this, the larger cytoplasmic lipid droplets induced in cultured rabbit liver cells are reported to constitute the thin 'floating fat' layer formed during centrifugation of the disrupted cells (Mackenzie et al., 1966). Recently, this 'floating fat' layer has been designated as storage triglyceride which contributes to the formation of secretory triglyceride and, moreover, to the hepatic free fatty acid pool (Bar-on et al., 1971).

The major purpose of our investigation was to study the morphology and composition of the floating, or supernatant, lipid-rich fraction observed at the top of rat liver homogenates after centrifugation. Our

\footnotetext{
* Present address: 250 Parsonage Street, Rocky Hill, Conn. 06067, U.S.A.
}

results show that this fraction is particulate and is composed predominantly of triglycerides and cholesterol esters. The particles in the fraction appear to be representative of the large cytoplasmic droplets observed in sections of liver cells.

\section{Methods}

\section{Preparation of lipid droplets}

Male Long Evans rats (250-320g) were maintained on NIH Sterilizable Rodent Diet, which was composed (w/w) of $24 \%$ (minimum) crude protein, $4 \%$ (minimum) crude fat, $3.5 \%$ (maximum) crude fibre and $8.6 \%$ (maximum) ash. During starvation studies the animals were allowed access to water ad libitum. The rats were killed between 08:00 and 10:00 h by cervical dislocation, the portal vein was incised and the liver perfused with chilled $0.15 \mathrm{M}-\mathrm{KCl}, \mathrm{pH} 7.0-7.4$, via the inferior vena cava, until all the lobes were visually exsanguinated. It was shown that perfusion did not significantly alter the yield (measured as triglyceride) or composition of the lipid droplet fraction. The livers were removed, freed from any attached adipose tissue and cut into small pieces, washed with the $\mathrm{KCl}$ solution, blotted, weighed and homogenized in 2-3vol. of the $\mathrm{KCl}$ solution in a Potter-Elvehjem homogenizer equipped with a loosefitting Teflon pestle. The homogenate was centrifuged at $17000 \mathrm{rev} . / \mathrm{min}$ for $100 \mathrm{~min}$ in a Sorvall RC2-B centrifuge with an SS-34 fixed-angle rotor. This centrifugation of the homogenate yielded a compact multi-layered pellet, a yellow-brown transparent infranatant and a floating white thin layer. For

Vol. 132 
compositional studies of the lipid droplets the floating layer was removed by aspiration with a syringe fitted with a long, blunted needle. It was important during this procedure not to agitate the tube, since the floating layer could be easily dispersed, at least partly, into the infranatant. When it was necessary more quantitatively to remove the lipid layer, cotton-tip applicators, previously washed in warm chloroform, were used to remove thoroughly any of the layer adhering to the wall of the centrifuge tube which could not be aspirated with the syringe.

\section{Analyses}

For each lipid-analysis experiment the floating layer derived from a pool of two rat livers was added to $100 \mathrm{ml}$ of chloroform-methanol $(2: 1, \mathrm{v} / \mathrm{v})$ containing $0.1 \%(w / v)$ butylated hydroxytoluene and extracted and washed under $\mathrm{N}_{2}$ as described by Folch et al. (1957). The extract, along with some insoluble tissue residue, was filtered through a medium-porosity sintered-glass funnel. The filtered extract was evaporated to dryness in vacuo at $35^{\circ} \mathrm{C}$ and stored under $\mathrm{N}_{2}$ at $-20^{\circ} \mathrm{C}$ in a small volume of chloroform. Separation of the lipid classes was made on columns of silicic acid (Bio-Sil A; 100-200 mesh; Bio-Rad Laboratories, Richmond, Calif., U.S.A.). The chromatographic column was made from a slurry of $10 \mathrm{~g}$ of the silicic acid in $n$-hexane; the final dimensions of the column were $1 \mathrm{~cm} \times 18.5 \mathrm{~cm}$. The chloroform extract (from storage) was evaporated to dryness under a stream of $\mathrm{N}_{2}$ and then suspended in 1-2 $\mathrm{ml}$ of hexane-benzene $(17: 3, v / v)$. The suspension was loaded on to the column and chromatographed for the different lipid classes by using the elution scheme of Barron \& Hanahan (1958). Fractions of eluate $(10 \mathrm{ml})$ were collected at a flow rate of $1.0 \mathrm{ml} / \mathrm{min}$. Lipid classes were identified in the eluate by t.l.c. of samples on silica-gel plates. The elution pattern of the experimental samples was in agreement with that of known lipid standards. The recovery of known standards from the column was $94-99 \%$. Various solvents and plates were used for t.l.c. Triglycerides and cholesterol esters were separated on silica gel $\mathbf{G}$ in benzene-hexane $(10: 1, \mathrm{v} / \mathrm{v})$; free fatty acids, monoglycerides, diglycerides and cholesterol were separated on silica gel $\mathbf{G}$ in hexane-diethyl etheracetic acid (50:50:1, by vol.); phospholipids were separated on silica gel $\mathbf{H}$ in chloroform-methanolacetic acid-water $(95: 35: 4: 3$, by vol.). Spots were detected by spraying the plates with $0.05 \%(w / v)$ of Rhodamine $6 \mathrm{G}$, followed by brief exposure to $\mathrm{I}_{2}$ and viewing under u.v. light. Lipid standards were purchased from the Hormel Institute, Austin, Minn., U.S.A. and Applied Science Laboratories, State College, Pa., U.S.A. All solvents used were reagent grade. Solvents used for column chromatography were redistilled before use.
Fatty acid analysis of triglyceride and cholesterol ester fractions was made by taking a sample of the appropriate combined column fractions and evaporating it to dryness under $\mathrm{N}_{2}$. Then $1 \mathrm{ml}$ of $\mathrm{BF}_{3}-$ methanol reagent $(14 \%$, w/v)-benzene-methanol ( $7: 6: 7$, by vol.) was added to the residue and the fatty acids were esterified under $\mathbf{N}_{2}$ in Pyrex screw-cap culture tubes with Teflon-lined caps immersed in boiling water (Morrison \& Smith, 1964). After $50 \mathrm{~min}$ the reaction mixture was cooled to room temperature, 2 vol. of pentane was added and the mixture was well agitated. Then 1 vol. of water was added, the mixture agitated again and the tubes centrifuged to separate the two phases rapidly. A portion of the upper, or pentane, phase was removed for analysis. The fatty acid methyl esters were analysed on a Packard 804 gas-liquid chromatograph with a flame-ionization detector. The glass coil column $[1.82 \mathrm{~m}(6 \mathrm{ft}) \times 4 \mathrm{~mm}$ inner diam.] contained $10 \%$ ethylene glycol succinate on Chromasorb W (AW; 80-100 mesh). The inlet, oven and detector temperatures were $188^{\circ}, 181^{\circ}$ and $205^{\circ} \mathrm{C}$ respectively. $\mathrm{N}_{2}$ was used as the carrier gas, at a flow rate of $60 \mathrm{ml} / \mathrm{min}$. Fatty acid methyl esters were identified by co-chromatography with standard fatty acid methyl esters.

Total triglyceride was determined by the method of Van Handel (1961) with tristearin (Hormel Institute) as a standard. In those experiments where it was only necessary to measure triglyceride plus one other component such as protein, a portion $(50-200 \mu \mathrm{l})$ of the chloroform extract was streaked on t.l.c. plates (250 $\mu \mathrm{m}$ thickness) of silica gel $\mathrm{G}$ and developed in benzene-hexane $(10: 1, \mathrm{v} / \mathrm{v})$. The band that corresponded to the triglyceride standard after brief exposure to $I_{2}$ vapour was scraped into glass-stoppered centrifuge tubes. The silica was extracted with $4 \times 3 \mathrm{ml}$ of warm chloroform, which recovered $96-105 \%$ of known amounts of tristearin. The chloroform washings were pooled, evaporated under a stream of $\mathrm{N}_{2}$ to $4 \mathrm{ml}$ and a sample was taken for the determination of total triglyceride as stated above.

Cholesterol was determined by the method of Abell et al. (1952). The volume of the modified Liebermann-Burchard reagent was decreased to $5 \mathrm{ml}$. Cholesterol purchased from a local supplier was recrystallized three times from ethanol before being employed as a standard. Phospholipid phosphorus was measured by using the Shin (1962) modification of the Fiske \& SubbaRow (1925) method. The amount of phospholipid was estimated by multiplying the amount of phospholipid phosphorus by 25 . For the measurement of protein in the lipid bodies the floating lipid layer was allowed to adhere to a glass stirring rod. The layer was removed from the rod by rinsing with $30-40 \mathrm{ml}$ of water and was centrifuged at $17000 \mathrm{rev} . / \mathrm{min}$ as stated above for $30 \mathrm{~min}$. This procedure was repeated again and the washed floating layer with $0.1-0.2 \mathrm{ml}$ of the lower clear phase was 
sonicated for $20 \mathrm{~s}$ at $60 \mathrm{~W}$ output with a $20 \mathrm{kHz}$ Sonifier Cell Disrupter (Branson Instruments, Inc., Melville, N.Y., U.S.A.). Protein in this sonicated suspension was determined by the method of Lowry et al. (1951), with crystalline bovine serum albumin (Pentex, Kankakee, Ill., U.S.A.) as standard. Protein determinations were also made on the lower (wash) phase to correct for non-particulate protein contamination arising from the soluble fraction of the liver. This correction usually accounted for $20-40 \%$ of the particulate protein. The presence of insoluble lipid in the sample used for protein assay caused a slight decrease in absorbance; the extent of this interference was determined by mixing various samples of the sonicated floating layer with known amounts of the serum albumin and measuring absorbance.

\section{Electron microscopy}

The floating layer was drawn up gently with a pipette and fixed in $4 \%(\mathrm{v} / \mathrm{v})$ acrolein and post-fixed with $2 \%(\mathrm{w} / \mathrm{v})$ osmium tetroxide (Sabatini et al., 1963). The insoluble sediment was rinsed with cold $0.9 \% \mathrm{NaCl}$, dehydrated with methanol and propylene oxide and finally embedded in Maraglass (Erlandson, 1964). Sections were cut with an LKB Ultratome I and stained with uranyl acetate and lead citrate (Reynolds, 1963). Thin sections were examined with an RCA EMU3H electron microscope.

A similar technique was used to obtain micrographs of the intact hepatocyte. The initial fixation step, however, was facilitated by perfusing the liver in situ with chilled $4 \%(w / v)$ acrolein.

\section{Results}

\section{Composition of the floating layer}

Analysis of the chloroform extract of the floating lipid layer by silicic acid column chromatography is reported in Table 1 . The layer was composed mainly of neutral lipids, predominantly as triglycerides. The total amount of triglyceride in the underlying or infranatant fraction was approximately $5 \%$ of that measured in the floating lipid layer. Cholesterol esters, the second most abundant lipid in the layer, were present at about one-tenth the amount of triglyceride. The cholesterol ester/triglyceride ratio did not change during two washings of the floating lipid layer. Only trace amounts of free, or unesterified, cholesterol and diglycerides were observed. Monoglycerides and free fatty acids were not detected. Small amounts of phospholipids were detected. When the phospholipid fraction was subjected to t.l.c., the major phospholipid found was phosphatidylcholine. The amount of protein in the twice-washed floating layer was also small, but this amount could partially reflect protein which was secondarily adsorbed from the soluble fraction. Thus the negative sedimentation property of the floating layer can be attributed to its very high neutral lipid/protein ratio.

While this work was in progress, Bar-on et al. (1971) isolated rat hepatic lipid droplets by overlayering the homogenate in $0.25 \mathrm{M}$-sucrose with water. After centrifugation, the lipid droplets appear at the top of the water layer and thereby permit the droplets to be sampled without sizeable contamination by the soluble fraction. When we prepared the droplets in this manner, followed by a single wash step, we obtained triglycerides, cholesterol esters and protein in proportions similar to those reported in Table 1.

The triglycerides of the lipid droplets consisted mainly of esters of linoleic acid (33.7\%), oleic acid $(30.2 \%)$ and palmitic acid $(24.7 \%)$. These acids contributed also the major esters for the cholesterol ester fraction: palmitic acid $(31.3 \%)$, oleic acid $(30.0 \%)$ and linoleic acid $(20.4 \%)$. The most dramatic difference in fatty acid composition between these two neutral-lipid classes was the nearly fivefold greater mole $\%$ of arachidonic acid in the cholesterol ester fraction $(1.4 \%$ versus $7.1 \%)$. Similar observations were made by Getz et al. (1961) who examined the fatty acid composition of the 'floating fatty supernatant' from the livers of rats of the Wistar strain.

The fatty acid composition of the triglyceride fraction of a liver homogenate which had had the floating lipid layer previously removed by centrifugation was remarkably similar to that of triglyceride of the floating lipid layer. When the pellet obtained after centrifugation of such a homogenate was vigorously re-homogenized with a loose- or tight-fitting pestle, the triglyceride in the supernatant fraction obtained after re-centrifugation was less than $5 \%$ of that in the original floating lipid layer. Thus it was unlikely that unreleased floating lipid in unbroken cells accounted

\section{Table 1. Composition of hepatic lipid droplets}

Composition is expressed as the average ratio to triglycerides $(\mathrm{g} / \mathrm{g})$. For experimental details see the Methods section. Numbers in parentheses represent the range of values obtained in four to six experiments.

$\begin{array}{ll}\text { Triglycerides } & 1.00 \\ \begin{array}{l}\text { Cholesterol esters (measured } \\ \text { as free cholesterol) }\end{array} & 0.10(0.08-0.11) \\ \text { Protein } & 0.032(0.025-0.036) \\ \text { Phospholipids } & 0.020(0.018-0.021) \\ \text { Diglycerides } & \text { Trace* } \\ \text { Monoglycerides } & \text { Not detected } \\ \text { Cholesterol (free) } & \text { Trace* } \\ \text { Free fatty acids } & \text { Not detected }\end{array}$

* Trace amounts were equivalent to a ratio $<0.01$. 
for the similarity between the fatty acid composition of triglyceride in the pellet and floating layer.

\section{Morphology}

As shown in Plates $1(b)$ and $1(c)$, electron micrographs of sections of samples of the floating lipid layer revealed numerous spherical osmiophilic bodies. No contamination by other hepatocyte organelles was seen. Most of the bodies had a diameter between 0.5 and $2 \mu \mathrm{m}$. Occasionally, much smaller bodies, about $50-200 \mathrm{~nm}(500-2000 \AA)$ in diameter, were observed in the floating lipid layer (Plate 1b). A thin limiting membrane appears to envelop each particle, regardless of size.

It was important during the isolation procedure to use relatively mild conditions in disrupting the hepatocyte. The use of a VirTis-type blade homogenizer, a sonicator, or a very tight-fitting pestle for the Potter-Elvehjem homogenizer resulted in fragmentation and coalescence of the lipid droplets. A common appearance for the droplets to take after severe mechanical treatment was long thin strands with attached small spherical 'buds'. Supernatant (soluble) protein from centrifuged liver homogenates appears to stabilize the droplets and easily facilitated their non-disruptive dispersion by homogenization or other mechanical means. Least disruption of the lipid droplets during the wash step was achieved by transferring the floating lipid layer from wash fluid to fresh water by utilizing its adhesion to a glass rod. Even so, some fragmentation of lipid bodies was evident in electron micrographs after two wash steps.

To provide evidence that the large lipid droplets isolated from rat liver were representative of a similar organelle observed in vivo, we examined the morphology of the normal rat hepatocyte. As shown in Plate 1(a), structures similar to those in the floating lipid layer were present in the cytoplasm of intact cells. Most of the large droplets observed in vivo did not stain as densely as those observed in vitro, but it is likely that this difference is an artifact caused by differences in fixation. The droplets in vivo occurred randomly in the cytoplasm and did not appear to be attached to any other organelle, although they were frequently adjacent to mitochondria. The droplets also appeared to be concentrated at the sinusoidal aspects of the liver. Occasionally, clusters of lipid droplets were observed near the periphery of sinusoidal cells. In some electron micrographs of the intact liver we noticed lipid droplets containing myelinlike figures. However, we did not observe any of the latter structures in the floating lipid layer.

\section{Effects of starvation}

Rats were starved for $72 \mathrm{~h}$ to examine the effects of starvation on the hepatic lipid droplet triglyceride.
The wet weight mass of the liver was decreased by 40-50\% under these conditions.

The amount of triglyceride in the lipid droplet fraction in fed rats was approx. $2 \mathrm{mg} / \mathrm{g}$ wet wt. and generally accounted for more than $50 \%$ of the total hepatic triglyceride. After starvation it dropped to approx. $50 \%$ of this value. On the other hand, the amount of triglyceride in the pellet (homogenate minus the lipid layer) was similar in starved and fed animals.

\section{Discussion}

The results of the present study suggest that membrane-bound droplets, rich in triglycerides and cholesterol esters, occur normally in the rat hepatocyte and are structurally independent of other cell organelles from which they can be separated by flotation. Other workers have produced histochemical evidence that these droplets are composed mainly of lipid and extraction of aldehyde-fixed hepatic tissue of rats with lipid solvents has been shown to cause disappearance of the droplets (Ashworth et al., 1966). Mackenzie et al. (1966) isolated the floating lipid layer from rabbit liver cells grown in culture and their compositional and micro-structural analysis is in agreement with the present study, except for the greater relative amount of cholesterol esters that we observed in the rat lipid droplet. The chemical composition of these lipid droplets is unlike that of the smaller 'liposomes', which have much higher amounts of protein and phospholipid according to Schlunk \& Lombardi (1967).

Except for the low amounts of phospholipid, the lipid droplets of our study have a composition not unlike that reported for rat chylomicrons (Redgrave, 1971). During fat absorption in the rat, osmiophilic droplets of $30-200 \mathrm{~nm}(300-2000 \AA)$ in diameter occur in the space of Disse and in vesicles near the periphery of the liver cell; such droplets are comparable with those observed in the intestinal lymphatics of fat-absorbing animals (Ashworth et al., 1966). However, the assumption that the lipid droplets might simply represent a fusion or re-assembly within the hepatocyte of chylomicrons appears unlikely in the light of recent observations. The hepatocyte culture studies of Mackenzie et al. (1962) revealed an absence of chylomicrons in the extracellular experimental culture media containing rabbit serum, despite the prolific formation of lipid droplets de novo within the cells. Felts \& Berry (1971) showed that chylomicron triglyceride fatty acids were not taken up or metabolized by liver parenchymal cells in amounts that were physiologically significant. It should also be pointed out that, like various serum lipoprotein classes (Lindgren \& Nichols, 1960), the lipid droplets might be heterogeneous in composition, but we have not studied this. 


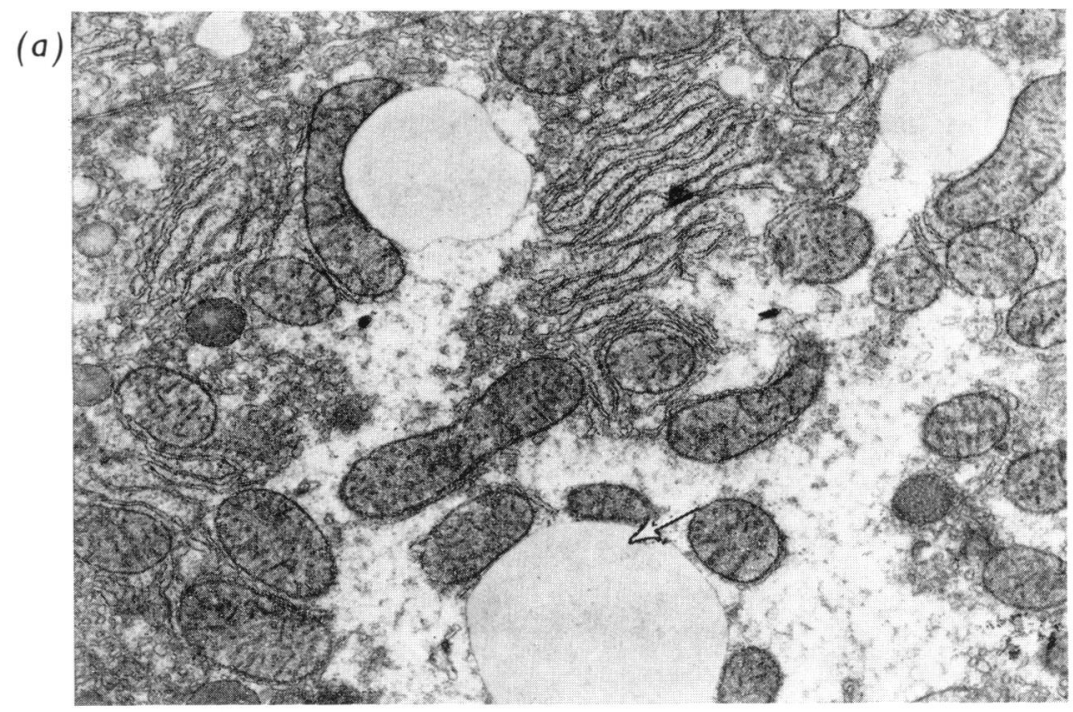

(b)

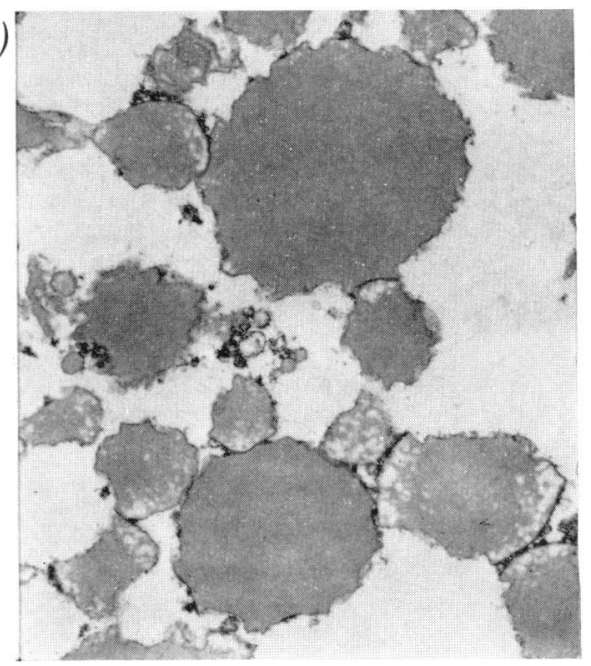

(c)

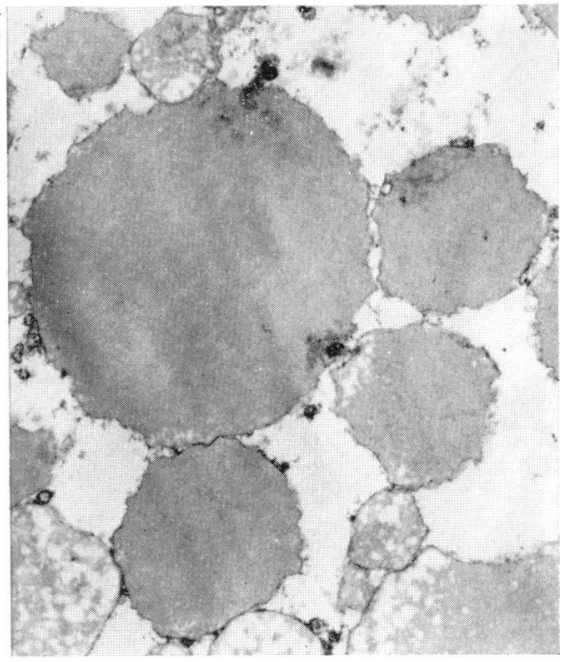

EXPLANATION OF PLATE I

Electron micrographs of hepatic lipid droplets

(a) Liver cell of rat fed ad libitum. The arrow indicates one of the large cytoplasmic lipid droplets. (b) and (c Hepatic osmiophilic droplets found in a sample of the floating lipid layer derived by centrifugation of rat liver homogenates. Magnification for $(a),(b)$ and $(c)$ was $\times 10000$. 
The slow turnover of glyceride precursors in the floating lipid layer (Stein \& Shapiro, 1959; Havel et al., 1962) has caused this fraction to be described as hepatic lipid depot. That variations in hepatic triglyceride, produced in the present study by starvation, were reflected in changes in concentration of triglyceride from lipid droplets and not in changes in the 'pellet' triglyceride content, would seem to support this concept. The decrease in triglyceride from lipid droplets during starvation is compatible with other results, showing that starved rats had a marked decrease in the number of lipid droplets observed in the hepatocyte (Ashworth et al., 1966).

The small amount of protein in the lipid droplets may be related to the thin osmiophilic limiting membrane seen in vivo and in vitro. It is possible that this coat consists of adsorbed protein, which confers hydrophilic properties to the droplet. Studies on milkfat globules, which also consist predominantly of neutral lipids, have shown that the globules are coated with a proteinaceous coat with enzyme activities suggestive of a cell-membrane origin (Dowben et al., 1967). This suggests that examination of the proteins of hepatic lipid droplets for microsomal enzymes, especially those participating in lipid deposition or breakdown, might be worthwhile. Rao \& Tarver (1970) have shown that there exists in the 'fat-rich supernatant fractions' of rat liver homogenates a system capable of incorporating leucine into protein in the absence of ribosomes.

The suggestions of Dr. L. Van Orden and the excellent assistance of Mrs. Sandra Gipson are gratefully acknowledged by us.

\section{References}

Abell, L. L., Levy, B. B., Brodie, B. B. \& Kendall, F. E. (1952) J. Biol. Chem. 195, 357-366

Ashworth, C. T., Leonard, J. S., Eigenbrodt, E. H. \& Wrightsman, F. J. (1966) J. Cell Biol. 31, 301-318

Baglio, C. M. \& Farber, E. (1965) J. Cell Biol. 27, 591601
Bar-on, H., Roheim, P. S., Stein, O. \& Stein, Y. (1971) Biochim. Biophys. Acta 248, 1-11

Barron, E. J. \& Hanahan, D. J. (1958) J. Biol. Chem. 231, 493-503

Dowben, R. M., Brunner, R. J. \& Philpott, D. E. (1967) Biochim. Biophys. Acta 135, 1-10

Erlandson, R. A. (1964) J. Cell Biol. 22, 704-709

Felts, J. M. \& Berry, M. N. (1971) Biochim. Biophys. Acta 231, 1-7

Fiske, C. H. \& Subbarow, Y. (1925) J. Biol. Chem. 66, 375-400

Folch, J., Lees, M. \& Sloane-Stanley, G. H. (1957) J. Biol. Chem. 226, 497-609

Getz, G. S., Bartley, W., Stirpe, F., Notton, B. M., Renshaw, A. \& Robinson, D. S. (1961) Biochem. J. 81, 214-220

Havel, R. J., Felts, J. M. \& Van Duyne, C. M. (1962) J. Lipid Res. 3, 297-307

Lindgren, F. T. \& Nichols, A. V. (1960) Plasma Proteins 2, 1-58

Lowry, O. H., Rosebrough, N. J., Farr, A. L. \& Randall, R. J. (1951) J. Biol. Chem. 193, 265-275

Mackenzie, C. G., Mackenzie, J. B. \& Reiss, O. K. (1962) J. Cell Biol. 14, 269-276

Mackenzie, C. G., Mackenzie, J. B., Reiss, O. K. \& Philpott, D. E. (1966) Biochemistry 5, 1454-1461

Morrison, W. R. \& Smith, L. M. (1964) J. Lipid Res. 5, 600-608

Novikoff, A. B. \& Essner, E. (1960) Amer. J. Med. 29, 102-131

Novikoff, A. B., Roheim, P. S. \& Quintana, N. (1964) Fed. Proc. Fed. Amer. Soc. Exp. Biol. 23, 126

Rao, K. R. \& Tarver, H. (1970) Arch. Biochem. Biophys. 141, 758-761

Redgrave, T. G. (1971) Aust. J. Exp. Biol. Med. Sci. 49, 209-224

Reynolds, E. S. (1963) J. Cell Biol. 17, 208-212

Rouiller, C. \& Jézéquel, A. M. (1963) Liver 1, 222-225

Sabatini, D. D., Bensch, K. \& Barrnett, R. J. (1963) J. Cell Biol. 17, 19-58

Schlunk, F. F. \& Lombardi, B. (1967) Lab. Invest. 17, 30-38

Shin, Y. S. (1962) Anal. Chem. 34, 1164-1166

Stein, O. \& Stein, Y. (1965) Isr. J. Med. Sci. 1, 378-388

Stein, O. \& Stein, Y. (1967) J. Cell Biol. 33, 319-339

Stein, Y. \& Shapiro, B. (1959) Amer. J. Physiol. 196, 1238-1241

Van Handel, E. (1961) Clin. Chem. 7, 249-251 